\title{
PERCEPCIÓN CIUDADANA DEL DESORDEN INFORMATIVO: SERVICIO PÚBLICO DE TELEVISIÓN Y ECOSISTEMA MEDIÁTICO
}

\author{
CITIZEN PERCEPTION OF NEWS DISORDER: PUBLIC TELEVISION SERVICE \\ AND MEDIA ECOSYSTEM
}

\author{
Concha Mateos \\ Universidad Rey Juan Carlos, España \\ concepcion.mateos@urjc.es \\ https://orcid.org/0000-0002-6805-0624
}

Cómo citar / Citation: Mateos, C. (2021) "Percepción ciudadana del desorden informativo: servicio público de televisión y ecosistema mediático". OBETS. Revista de Ciencias Sociales, 16(2): 417-434. https://doi.org/10.14198/OBETS2021.16.2.12

\section{(C) 2021 Concha Mateos}

Este es un artículo de acceso abierto distribuido bajo los términos de la licencia de uso y distribución Creative Commons Reconocimiento 4.0 Internacional (CC BY 4.0) https://creativecommons.org/licenses/by/4.0/deed.es

Recibido: 17/06/20. Aceptado: 31/12/20

\section{Resumen}

La percepción del público sobre el servicio público televisivo (SPT) constituye un objeto de estudio tradicionalmente desatendido, mientras trabajos previos señalan que el SPT precisa ser rediseñado atendiendo a esa percepción.

Esta investigación lo aborda con el objetivo de conocer cómo percibe el público el SPT y qué función espera que cumpla dentro del conjunto del sistema de medios. El método aplicado opera a partir de datos producidos en grupos de discusión. Se sigue un procedimiento de análisis del discurso desde una perspectiva sociolingüística orientada por la economía de los intercambios lingüísticos de Bourdieu. Se articula un dispositivo multidisciplinar para la observación del discurso, combinando diversas herramientas conceptuales: consenso operativo, turno de palabra, silencio y movimientos de la conversación, tomados de Goffman (1967 y 1956); dinámicas del mercado lingüístico, de Bourdieu (2016 [1982]); sentidos transversales, de Conde (Conde, 2010); isotopía y campos conceptuales, de la Semántica Interpretativa; elección de persona gramatical y actitudes

\section{Abstract}

Public perception of the public television service (SPT) is a traditionally neglected field. As a result, there are research studies that indicate the SPT needs to be redesigned to build a new relationship with the audience. Reinvention is essential for the SPT to survive, warns Freedman (2016b), who adds, "audiences deserved to be treated more than as just consumers". Cullinane (2017) points out that an SPT needs legitimacy in a democracy and legitimacy comes from public recognition.

Regarding the public, there are studies that explore experiences with new forms of audience participation: Keinone and Klein (2017) via multiplatforms and Vanhaeght (2019), which studies the experience of integrating the public in the production of the programme, "The Monitor", on the German public television channel, NPO. This author observed that studies are still needed to find out how the audience perceives these results.

This research specifically studies public discourse on the perceived function of the SPT in the middle of the

1 Este artículo cierra una serie de dicada al estudio de la percepción del SPT firmados por la autora junto a María Lamuedra y Manuel Antonio Borullón. En particular, este trabajo pretende sentar una referencia para la observación futura del impacto que, sobre esa percepción, vaya generando el desarrollo del fenómeno fake que implosiona a partir del año 2016, cuando se cierra la recogida de discursos sobre los que trabaja esta investigación. 
proposicionales, de la Gramática; y figuras del lenguaje, de la Retórica.

Se acota la temporalización al año 2016, para extraer un cuadro sincrónico que pueda servir de referencia sobre la visión SPT antes de la explosión de fenómeno de las fake news agudizado a partir de las elecciones presidenciales estadounidenses de ese año.

Los resultados ratifican observaciones de estudios previos y también introducen parámetros nuevos en la concepción popular del SPT, que invitan a una renovación de la concepción académica del servicio.

La preocupación por la fiabilidad o/y calidad de los contenidos de los medios no se refiere ya únicamente el producto final, hay perfiles de público sensible a la calidad del proceso de producción. La demanda de reconfiguración del SPT ya no se formula en términos de escala cuantitativa: más pluralismo, más acceso, más representación de voces o cultura, más diversidad, más inclusión. Se ha activado también una demanda de otro concepto de lo público. No solo al margen de los partidos políticos, sino como exigencia de otro concepto de ciudadanía y de gestión de lo común. Otra forma de ejercer la propiedad ciudadana del SPT se considera pendiente

Palabras clave: Televisión pública; servicio público; TVE; desorden informativo; regulación; confiabilidad; grupos de discusión; análisis del discurso.

Thus, the old idea of Walter Lippmann applies: a society that lacks the tools to control lies in its public communication forums cannot consider itself free (Kalsnes, 2018, sn).

In this context, public discourse produces isotopies around various shared perceptions. I have highlighted six of these.

Isotopy of the opaque issuer. The public notes that decision-makers in the media often remain in the shadows: Who is that unknown decision maker? a participant asks. Others talk about something anonymous, not physical, with no visible head.

Isotopy of hidden interests. They talk about "economic interests behind them", of puppets, of intermediaries who obey, of hiding their true identities and of disguise. And they frequently use adverbs to qualify the actions of the issuers: e.g. apparently, supposedly.

Isotopy of deception. There is a perception that lies circulate freely through the ecosystem. They are expressed in many different ways. They use verbs like: manipulate, camouflage, hide, contaminate, plant stories, distract. They qualify the media content as: a show, something chosen, controlled, very measured, unreal. Also, they choose particular expressions to describe what the media do: they do not let you see what is really happening, they tiptoe around a subject.

Isotopy of a state of war and being hunted. They often describe the media environment in terms of a threat, war, or an attack. They talk about being invaded, of being very media ecosystem. The research is based on data produced from discussion groups. It describes the procedure applied to analysis of the discourse in detail. This analysis was conducted from a sociolinguistic perspective, with a methodological design based on Bourdieu's economics of linguistic exchanges.

One of the central analytical tools is isotopy: the use of terms pertaining to the same or similar references. By discovering isotopies, the shared meaning across a set of discourses can be revealed.

Isotopies point to redundancies, convergences, shared visions: "a permanent reference base, by virtue of which the discourse becomes coherent or, in other words, speaks of the same thing" Bueno and Blanco (1980: 36).

Thus, this analysis looks at the discourse of the groups to identify what role the SPT plays, according to the public, in the midst of a media ecosystem of increased competition (Levi, 2020:15); when traditional media have lost their ability to control the topics that dominate the attention of the audience every day. (Elías, 2018; Bennett \& Linvingston, 2918; Waisbord, 2018).

This is an ecosystem where numerous agents individual, institutional, public, and private - all compete at the same time to dominate the agenda. And, in the midst of this dynamic, fake news can spread faster than real news (Vosoughi et al, 2018); such that a high quality, informative article has no guarantee of enjoying any advantage in the possibility of being disseminated. (Shao et al., 2018: 2).

careful, of being bombarded or trapped; or you have to find places or programmes to take refuge.

Isotopy of overload. The media ecosystem is represented as a saturated territory where you have to have certain skills to avoid danger or risk or to be able to move. They say there are millions of opinion-makers, that you do not know where to go to choose, there is an excess of information, that they grab your attention. They talk about the difficulty of having sufficient capacity to absorb, to handle, to screen, to summarise. And also, of the need to have a medium to act as a reference in the midst of everything.

Isotopy of lack of quality. There are public profiles that find a lack of quality in the product features: it is not objective, impartial, or real. While other profiles focus on a lack of quality in the production process: there is little checking, it is a perversion, there are no barriers, information is selectively chosen, sources are not traceable, or it is all a set-up.

The analysis notes that this division between focusing on the product and the process coincides with another division: between belief and a lack of belief that objectivity is possible. And, in turn, both are based on two expressive styles. Speakers who prioritise a focus on the product and belief in objectivity often use the passive voice: "The media was informed" or "news was received". The other expression profile opts for the active format: I found out. Finally, this process-based focus with an active voice also coincides with those who demand that procedural norms be observed, that the media contribute to democracy and guarantee participation, among others. 
The six isotopies found in the public discourse of the media ecosystem and the role of the SPT show a perception of structural information disorder in the system, which induces feelings of insecurity and mistrust.

The media in general, and the SPT in particular, are perceived as a function of democratic power, but poor and unsatisfactory in practice. This tension has been noted by authors such as Fenton (2018).

Citizen discourse discusses aspects that renew the traditional academic frameworks for understanding the SPT, while sometimes confirming and expanding upon conclusions from more recent studies. The concern for the reliability and/or quality of media content does not refer only to the final product, as there are public profiles sensitive to the production process quality. Agreement with Larsen (2014:74).

The demand for reconfiguration of the SPT is no longer formulated in terms of quantity - more pluralism, access, representation of different voices or culture, diversity, and more inclusion, etc - but a demand for another concept of what is "public". And not only outside the political parties, but also as a requirement of another concept of citizenship and management of what is common. Another way of exercising public ownership of the SPT is considered as pending.
In particular, information professionals are considered as captive subjects of the business or political structure, as a limiting element of their principles and professional skills.

As a result of this, the main demands are concerned with increasing transparency in the media production structure, by developing a legal framework for the protection of professional independence and the regulation of quality standards, as measures to guarantee that the media content gains in reliability.

Closely linked to reliability, there is also a demand that the media, particularly the SPT, expand the information they provide about themselves to the public as broadcasters as well as regarding the professionals working in them; i.e. that they provide information on the selection processes and criteria that professionals have to overcome to work in the SPT. Also, information about their training and professional careers, both creative and production professionals, as well as managers. This demand should be applied to both public and private media.

Keywords: Public television; public service; TVE; information disorder; fake news; regulation; reliability; media ecosystem; discussion groups; isotope discourse analysis.
El servicio público de televisión (en adelante SPT) se acerca a cumplir su primer siglo de existencia -la compañía que dio lugar a la BBC fue creada en 1922 aunque no fue hasta 1926 cuando se reconvirtió en entidad pública (Freedman, 2016a:16-17; Arbona, 1994:87) - en un mundo muy distinto al que lo vio nacer.

Entre los años 60s y 80s del siglo XX, cadena de rupturas de los monopolios audiovisuales originarios. En los años 90s, multiplicación de canales por cable y satélite. En junio de 2000, conferencia internacional "Public Broadcasting Beyond 2000" en Canadá: los expertos cierran el siglo con la clara conciencia de que el SPT necesita ser reinventado (Collins et al.: 2001).

Luego, se consolida Internet. Desde la aparición de la televisión, ninguna novedad había supuesto una transformación tan global de la comunicación social (Raboy, 2006: 290). Empiezan los estudios del cambio profundo en los patrones de consumo mediático (Fenton, 2010a, 2010b). Las audiencias ya no esperan sentadas el horario de emisión de sus programas favoritos, los jóvenes especialmente buscan información por vías no tradicionales, los datos ratifican que los niveles de credibilidad de los medios en los años 80s no se vuelven a alcanzar (Pew Research Center, 2005:41).
Llegan entonces las redes sociales y producen un giro radical en la forma en que las personas acceden e interactúan con la información (Jacques et al. 2019: 1; Shao et al, 2018; Ofcom, 2018: 7 ss.; Bakshy et al., 2015:1130; Peñamarín, 2014:105; Holton, 2013: 620; Fenton, 2010b: 41). La mediación tradicional se diluye a favor de nuevos agentes comunicativos en el ecosistema mediático (Freedman, 2016a: 8) y los medios "poderosos" tradicionales pierden influencia (Elías, 2018; Bennett \& Linvingston, 2918; Waisbord, 2018). Cada unidad de la multitud (persona, anónimo o institución) ejerce de fuente informativa, sin mediación, con canal propio. La competencia entre actores en el foro público se intensifica (Levi, 2019:15). Incluso dentro del sector del servicio público, hay agentes que no son televisiones pero producen también contenidos informativos en vídeo, los muesos entre otros (Freedman, 2016a: 157). Y además, el mercado de contenidos adopta una escala global (Ofcom, 2018:1014; Gardner, 2017: 8-10).

El rastro de las interacciones de todos estos agentes y sus públicos en ese mercado global genera minas de información que, procesadas por logaritmos opacos (Peirano, 2019: 23-24; Fenton, 2018: 33; Lazer et al. 2018:1095; Bakshy, 2015) determinan la pirámide de 
visibilidad en la que se ordenan los contenidos: qué es lo más buscado. Una multitud dispersa sustituye ahora a los editores de informativos (Lee-Wright, 2010:72) para decidir lo que aparece en la primera página del ecosistema.

En esa dinámica, las noticias falsas se difunden con más rapidez que las verdaderas (Vosoughi et al, 2018), la alta calidad de una pieza informativa no asegura ninguna ventaja en sus posibilidades de difusión (Shao et al., 2018: 2).

El Consejo de Europa ha sostenido (Nissen, 2006: 21-23) que las tres funciones del tradicional "pacto con la sociedad" del SPT se mantienen, aunque varíen los medios para desarrollarlas: 1) servir a la diversidad cultural, 2) fomentar la cohesión y 3) sustentar la cultura y dinámica democráticas. Y también se mantiene la línea de trabajos que abogan por proteger al SPT de presiones económicas y políticas para permitir que cumpla sus funciones (Cullinane, 2017; Arriaza et. al, 2015; Cushion, 2012; Dahlgren, 2009; Humphreys, 2008; Moe, 2008; Steemers, 2001; Blumler, 1993). Pero no han cesado las presiones que le dificultan atender al interés general:

1. Una audiencia ampliamente saturada (Holton et al., 2013), en un contexto caracterizado sobre todo por la abundancia (Raboy, 2006: 298) donde la audiencia del SPT se reduce en medio de un proceso general de fragmentación (Feedman, 2016a:33; Bourdon et al, 2019:1).

2. Largo periodo de políticas de carácter neoliberal que han impuesto recortes de fondos públicos (Sehl, Cornia \& Nielsen, 2016:7; Padovani \& Tracey, 2003:137-138) y un modelo de SPT preferentemente orientado al mercado (Bardoel \& d'Haenens, 2008: 251,252; Garnham, 1983:23), con las consiguientes constricciones que ese mercado impone (Fenton, 2010b:5051), forzándole a programaciones diseñadas para luchar por la audiencia (Cullinane, 2017). En general, la dependencia publicitaria (Norcicity, 2011 y 2016) y los recortes presupuestarios públicos son tendencia consolidada desde los 80 s y los 90s del siglo XX.

3. Un proceso creciente de ampliación del marco de competencia -globalización- (Ofcom, 2018: 14-15), ante el que Conway nos pregunta: "Why should a national government, for example, provide public funding for a broadcaster that viewers will not watch because they prefer slicker programming from somewhere else?" (Conway, 2009:73).

Estas presiones erosionaron mucho el valor distintivo del SPT (Lamuedra, Mateos y Broullón, 2020 y 2019; Padovani \& Tracey, 2003:140). Sin especificidad, el SPT no logra ser reconocido por la sociedad a la que sirve y eso le impide alcanzar legitimidad (Campos; Túñez y Rodríguez, 2018). Y, si se añade a ese contexto la prosperidad del flujo de información viciada (Levi, 2020: 38-41), el resultado es un cuadro crítico para las sociedades democráticas, que necesitan, como se ha señalado largamente, disponer de noticias para ejercer sus derechos y deberes.

Se reactualiza la vieja idea de Walter Lippmann: una sociedad que carezca de las herramientas para controlar las mentiras en sus foros de comunicación pública no puede considerarse a sí misma libre (Kalsnes, 2018, sn).

Un siglo más tarde de que Lippmann expusiese su idea, este trabajo se pregunta por la función que el público atribuye al SPT en este nuevo ecosistema mediático.

\section{JUSTIFICACIÓN}

La visión del público ha sido la menos explorada y la menos tenida en cuenta en los diseños políticos del SPT. En parte por la originaria perspectiva paternalista que dominó sus diseños iniciales (Arbona, 1994, 87-88). Previamente a la renovación obligatoria de sus cartas reales cada 10 años, la BBC realiza un proceso amplio de consultas públicas. El último se cerró en 2016 (DCMS, 2016; DCMS, 2015) para renovar al Carta Real que regía desde 2007. También la primera legislatura del gobierno de José Luis Rodríguez Zapatero en España (2004-2008) encargó en su día un proceso de consulta a expertos (Zallo, 2010) para fundamentar la redacción del nuevo estatuto del SPT aprobado en 2006.

La consulta de la percepción ciudadana en profundidad, en el caso de la BBC, es una pauta regular (Ipsos Mori, 2017:1); en el caso de España, una rareza. Como señala Meijer (2012: 754-755) refiriéndose al periodismo, la apreciación que tiene la audiencia ha arrastrado un déficit de atención investigadora. Esta 
falta de atención agrava el problema que Cullinane (2017) observa: la legitimidad que está pendiente para el SPT es la legitimidad emanada desde el público.

Los patrones de consumo sí reciben la atención en estudios periódicos. Los datos de la Pew Research Center (2018:14) revelan que las cadenas de televisión pública en la mayor parte de Europa ratifican su rol de fuentes de referencia y son las más utilizadas por la ciudanía para informarse.

En Estados Unidos, donde la televisión pública ha desarrollado tradicionalmente un rol marginal, los datos de Gallup sobre consumo y credibilidad (Brenan, 2019) indican un robustecimiento: de 1998 a 2019 desciende acusadamente el consumo informativo de las cadenas privadas (del $75 \%$ al $40 \%$, los programas de noticias nocturnos de ABC, CBS y NBC; y del 38\% al 23\%, la CNN) mientras se mantiene el de la cadena pública, (crece, de 28\% a 30\%) que, aunque es más bajo, goza de mayor grado de confiabilidad (59\% frente al $54 \%$ de ABC, CBS o NBC, el $48 \%$ de CNN y $43 \%$ de FOX news).

En España, el primer canal de televisión pública, TVE, se mantiene como medio preferente para información política en periodos electorales (CIS, 2019: 6). Tras la primera semana de medidas de control de movilidad social por la crisis del Covid19 (23 al 29 de marzo 2020), en el primer informe de OFCOM sobre fuentes informativas utilizadas por la población para informarse sobre esta crisis, el $82 \%$ señala a la BBC como preferencia.

La responsabilidad del Estado para ordenar el sistema mediático es altamente reconocida en España. Reuters (Newman et al., 2018:19) muestra que España es el país donde más personas suscriben la idea de que el Gobierno debiera hacer más para asegurar la diferenciación entre lo que es real y lo que es fake en internet. Lo apoyan el $72 \%$, por encima del $61 \%$ que lo hace en Reino Unido o Francia, y lejos del 41\% que lo hacen en EEUU.

Vamos a explorar qué razonamientos hay detrás de estas cifras. El interés en realizar este estudio reside en que los expertos han señalado entre los diez primeros retos del SPT el de redefinir su función (Campos; Túñez y Rodríguez, 2018:9). Según Freedman (2016b) reinventarse no es una opción, es la única opción para la supervivencia.
Sin reinvención no habrá legitimidad y la legitimidad es una exigencia democrática, señala Cullinane (2017: 107), que también indica que la reinvención requiere ante todo que el SPT cambie su mentalidad sobre la audiencia. La vieja asignatura pendiente que señalaba Garnham (1983:23): ser capaces de adoptar otra visión sobre la audiencia. El aviso de Freedman (2016b): "audiences deserved to be treated more than as just consumers".

Atentos al público, Keinone y Klein (2017) han explorado su percepción sobre ciertas formas de participación e interactividad mediante prácticas en multiplataformas y proponen el neologismo de Soci(etal) TV para referirse a esta modalidad.

Vanhaeght (2019) ha estudiado la experiencia de integrar al público en la producción del programa The Monitor de la cadena de televisión pública alemana NPO y confirma que los resultados acercan la agenda informativa al interés ciudadano, pero termina señalando que aún faltan estudios para conocer cómo percibe la audiencia estos resultados.

Todo ello fundamenta esta propuesta de investigar lo que espera la gente del SPT. Un trabajo que se traza desde los objetivos de las ciencias menores o nómadas, según Jesús Ibáñez, citado por Gordo y Serrano (2008: XVIII): "orientarse hacia dicha transformación y perseguir objetivos emancipatorios en un intento de comprender lo social a través de un proceso de reconsideración, desvelamiento y cuestionamiento de intereses, ideologías y sentidos atribuidos a los fenómenos sociales".

\section{MÉTODO}

\subsection{Objetivo}

Freedman (2016a: 24) señala que la misión y la posición del SPT se define en relación con los demás agentes del sistema de medios, no de forma aislada. Por ello, el objetivo principal de este trabajo es conocer la percepción del público sobre el sentido que tiene el SPT dentro del ecosistema mediático. Se busca la respuesta a las preguntas:

1. ¿Qué identidad y función se le atribuye al SPT dentro del sistema de medios?

2. ¿Qué valores, requisitos y rasgos distintivos se le asignan en ese contexto? 


\subsection{Perspectiva}

Este trabajo estudia el discurso del público sobre el SPT desde una orientación informada por la teoría sociolingüística, en la tradición del pensamiento de Halliday (2013 [1979]), y desde el marco operativo de interpretación de la economía de los intercambios lingüísticos de Bourdieu (2016 [1982]).

El estudio asume que el uso del lenguaje modela socialmente y está modelado también socialmente (Bourdieu, 2016: 11-17; Conde, 2010: 9), construye sistemas de conocimiento y creencias (Fairlough, 1993:134; 2006:62 ss.) y que los hablantes dejan rastros de ese modelo en sus actos de habla.

Se observará el uso del código lingüístico (uso semántico, gramático y pragmático de la lengua), el habitus de los hablantes (disposiciones, capacidades e intereses que maneja cada hablante y que se han elaborado socialmente en su historia personal de interacciones previas) y la dinámica del mercado lingüístico particular que toma forma en sus conversaciones (Bourdieu, 2016:11-12).

Sabemos que "el objetivo del análisis del discurso es dar cuenta de la dispersión de enunciados existente en un campo discursivo" (Gordo y Serrano: 2008: 16). El campo discursivo que aquí nos ocupa es el de las funciones que el público considera que debe desempeñar el SPT dentro del ecosistema mediático. Buscamos así identificar las creencias y los problemas que delimitan la conversación social del público sobre ese asunto, en el momento previo a la implosión de una nueva versión del fenómeno fake news que se dispara a partir de las elecciones presidenciales de 2016 (Elías, 2018) en Estados Unidos. De este modo, nos dotaremos de un cuadro de referencia para observar el impacto que el fenómeno tiene en la percepción ciudadana del SPT en fases futuras.

\subsection{Objeto de estudio}

El objeto intelectual de estudio es un campo discursivo. El público no es una masa homogénea en preferencias. Tenemos que asumir que ese campo discursivo que pretendemos estudiar es un campo discursivo en discusión, un marco atravesado de tensiones.

No se persigue aquí el retrato estadístico de esas preferencias. Se busca conocer el marco de enunciación en el que esas diferentes posiciones negocian el sentido que le atribuyen al SPT (Goffman 1967, 1956). Identificar lo que les permite discutir.

El objeto investigado es un fenómeno de producción social de significado y la perspectiva metodológica seguida se inspira en el interaccionismo simbólico, por eso el objeto se estudia a través de la conversación, y utilizando una paleta de herramientas multidisciplinares.

Nos interesamos por la conversación porque en las conversaciones aflora y se regula tanto lo que se opina, como lo que se piensa que opinan otros o lo que se considera que es aceptable/inaceptable opinar; es una forma de actualizar la cultura del grupo (Goffman, 1956:94). Observaremos pues, no tanto la opinión expresada por cada persona, sino la norma asumida en la forma de expresar esa opinión (habitus lingüístico en términos de Bourdieu).

Abordaremos el objeto de estudio en un contexto específico, un país donde el SPT ha tenido un liderazgo mediático sostenido, España. La razón es que esta investigación adopta una perspectiva crítica de intervención sobre la realidad: persigue un conocimiento situado (Haraway, 1995) que pueda ser útil para informar políticas locales en el área geográfica que estudiada.

\subsection{Conformación de la muestra}

Para producir los datos, se recurre a un método cualitativo dialógico: los grupos de discusión. El objeto material de estudio van a ser las conversaciones de cinco grupos sociales de diferentes perfiles, definidos en función del tipo de relación que las personas mantienen con el SPT, así la investigación cumple el requisito de ajustar el diseño a la primacía de los objetivos (Conde, 2010:9; Gordo y Serrano: 2008: XVII y 60-61). Se diseñaron inicialmente tres grupos (G1, G2, G3), de entre 6 y 8 participantes. Se advirtió un déficit de presencia de posiciones ideológicas conservadoras y se añadió entonces un grupo (G4) de perfil conservador. Una vez realizado, se advirtió una sobrerrepresentación de discurso desde posiciones de nivel formativo superior y se realizó por ello otro grupo más con personas sin estudios superiores (G5). Tras los cinco grupos, se constató una saturación discursiva suficiente (Callejo, 2000: 77): se estabiliza el patrón de ítems con que los participantes responden a qué servicio se espera del SPT, con qué valores y para qué funciones. 
Tabla I.

\begin{tabular}{l|l|l|}
\hline G1 - ACTIVISTAS & \multicolumn{1}{c}{ Rescripción } & \multicolumn{1}{c}{ Realivación } \\
\hline G2 - SERVICIO PÚBLICO CULTURA & Tienen experiencia en sector público cultural & $\begin{array}{l}\text { La Laguna } \\
17 / 03 / 2015\end{array}$ \\
\hline G3 - INTERNAUTAS & & $\begin{array}{l}\text { Madrid } \\
07 / 03 / 2015\end{array}$ \\
\hline G4 - CONSERVADOR & Consumen medios a través de internet & $27 / 2015$ \\
\hline G5 - SIN ESTUDIOS SUPERIORES & Contrapeso sobrerepresentación universitaria & $\begin{array}{l}\text { Sevilla } \\
29 / 05 / 2015\end{array}$ \\
\hline
\end{tabular}

Grupos de discusión.

El reclutamiento se ha realizado siguiendo el planteamiento de red polímera de Jesús Ibáñez (2015: 422): partiendo de contactos de una red natural preexistente del equipo de investigación pero evitando el vínculo inmediato y rompiendo el vínculo mediante la intervención mediadora de un profesional. Dentro de cada grupo (formado con criterio de homogeneidad) se ha buscado la diversidad sociológica (criterio de heterogeneidad) por edad, género y perfil profesional. Los grupos se han celebrado dentro del marco del Proyecto de investigación "Dinámicas de relación ante el cambio social: contextos, contenidos, productores, público y produsuarios en las noticias de TVE e YLE" (CSO2013-45470-R).

\subsection{Producción de datos y herramientas de análisis}

Las reuniones se moderaron con estilo no directivo (Gutiérrez, 2014: 47) para favorecer que la emergencia o ausencia de temas pudiera resultar significativa. Se iniciaron planteando una propuesta de diálogo abierta sobre el asunto "medios de comunicación". En cada grupo participó un observador recogiendo referencias de las interacciones no verbales.

Transcritas las conversaciones, se han analizado los discursos mediante una secuencia recursiva ${ }^{1}$ de tres estaciones:

1. Observar por separado las condiciones de producción del discurso de cada grupo: la economía de la atención y los procesos de

1 De idas y vueltas sucesivas entre el conjunto y el detalle, el código y el acto de habla (Conde, 2010:118). puntuación -recorte y organización de los hechos(Ibáñez, 2015: 402). En resultados, un epígrafe describe los distintos estilos de interacción y las dinámica de mercado lingüístico (Bourdieu, 2016) que ha desarrollado cada grupo.

2. Identificar el mapa de relevancia temática del conjunto de discursos por recurrencia: temas que emergen en varios grupos. Los criterios para esta selección son: 1) cuantitativo, presencia al menos en más de la mitad de los grupos y tratado al menos por más de la mitad de las personas de cada grupo; y 2) cualitativo, temas que retroalimentan las conversaciones, generan hilo conversacional: por tensión, confrontación, reapropiación cruzada de términos, reformulaciones, consensos. Herramientas aplicadas para esta fase: consenso operativo, turno de palabra, silencio y movimientos de la conversación de Goffman (1967 y 1956); y dinámicas del mercado lingüístico de Bourdieu (2016 [1982]). ${ }^{2}$

3. Cruzar los diversos discursos para localizar sentidos transversales al conjunto (Conde, 2010: 110).

Herramientas lingüísticas aplicadas en esta fase: la isotopía y los campos conceptuales de la Semántica Interpretativa (Rastier, 2005; Martínez, 2003; X), los usos de pragmagramática (Blas, 2004; Briz, 2001; Gallardo, 1996); la elección de persona gramatical y actitudes proposicionales (Fernández, 1995) de la

2 En la tabla, un código de marcas dará información sobre la presencia de estos fenómenos en la conversación. 
Gramática. De la Retórica se han tomado las figuras del lenguaje, principalmente transferencias de significado mediante metáforas y metonimias.

\section{RESULTADOS}

\subsection{Perfiles de interacción y dinámicas de mercado lingüístico}

Cada uno de los grupos desarrolla una dinámica de interacción diferente. Sabemos que todo acto de habla es una coyuntura (Bourdieu, 2016:12) que interfiere en el sentido de lo dicho. Las formas de sucesión/robo/ interrupción etc. de los turnos de palabra modelan ese sentido y jerarquizan el interés de los temas.

1. G1-Activistas es con diferencia el grupo en el que más se articula un consenso explícito y operativo (se esboza un proyecto de SPT entre distintas voces). Son constantes las expresiones como: "sí, yo también", "como comentaba ella", "es lo que tú decías -que tenías mucha razón-...". Los hablantes se respaldan mutuamente en el desarrollo temático de las conversaciones (Blas, 2004,164), asumen valoraciones de otros, las completan, retoman términos ya empleados por otra persona, principalmente en lo que respecta a cómo están funcionando los medios y qué se puede o se debe hacer con el SPT. El respaldo se da en diversas direcciones y desde varios sujetos. Este es el único grupo que llega a utilizar el "nosotros" como sujeto de una acción constructiva de SPT. Lo hace en la segunda mitad de la conversación, en la que, a la vez que van desapareciendo quejas o descripciones "doloridas" de lo que hay, va ocupando más espacio la conversación sobre cómo quisieran que fuera el SPT. Defienden con detalles una refundación del concepto de SPT gestionado "por la gente" y señalan por tanto la necesidad de que el público asuma su responsabilidad en el desarrollo del SPT.

2. Diego: "nosotros somos los primeros que tenemos que aprender"

Carlos "tenemos que constituir grupos donde la gente participe. Donde la gente decidamos"

Javier: "Además esa responsabilidad... la... la tenemos que asumir en todos los niveles. Económico también..."
Diego: "Es que incluso podríamos, verás ... (...) Hay una cosa que... que me da vueltas hace tiempo que es... realmente ... Creo que ahora mismo tenemos mecanismos mucho más democráticos como de crowdfunding tal para... para... para ... para decidir los ciudadanos, dónde queremos... qué... qué queremos apoyar realmente con los recursos que tengamos. Creo que los medios... en general -de esto no sería solamente para periodistas o para el tema del debate público- pero en general creo que para eso, para investigación, para tal (...) público pero directamente desde los ciudadanos".

3. G2-ServicioPúblicotambién presentaconvergencias sobre la idea de que el SPT es necesario como garantía (estructural) de determinada condición democrática de la sociedad. Pero la economía de la atención en este grupo no se administra, como en G1-Activistas, desde el foco de "nuestra responsabilidad y nuestra iniciativa respecto al SPT" sino desde la crítica de lo que debería ser y no es. Ese es el eje temático central en este grupo. Y sí aparecen enunciados empleando la primera persona del plural, especialmente en el último tercio de la conversación, cuando ya se han "desvelado" las posiciones discursivas de cada persona, o, en términos de Bourdieu, cuando ya se han fijado los valores discursivos que cotizan en ese mercado lingüístico. Esta intervención de Iñigo, por ejemplo, en el minuto (1:38:27) deja constancia de que él ya ha apreciado que la coyuntura (contexto) le permite hablar por los demás hasta cierto punto: Iñigo: "somos más críticos con la televisión pública pero precisamente porque es pública. Tienes más exigencia a lo que es tuyo o lo que consideras tuyo, que hacia el canal de Berlusconi". Ese mercado se ha clarificado porque han abundado los reconocimientos mutuos, del tipo: Alicia: "Yo creo que has dado en clave", Marichu: "... estoy de acuerdo contigo en que es el NODO". Iñigo: "Bueno, para mí la verdad coincido con ella... (en las críticas a la pública)". Iñigo: "es lo que tú dices...". Estos respaldos discursivos cruzados versan principalmente sobre el tema del control político y económico sobre los medios y la importancia estructural del SPT en la sociedad democrática. 
4. En G3-Internautas se manifiestan discrepancias expresas respecto a lo que esperan y aspiran que sea SPT pero este tema no ocupa apenas espacio en su conversación; es el grupo que menos habla sobre el SPT, o sobre la calidad de los contenidos y la información que proporcionan los medios. Su eje temático central es "cómo usar los medios". La práctica informativa se concibe aquí como acción del sujeto: las personas se informan ("yo es que me informo realmente" "busco en..."). A diferencia de la imagen de sujeto receptor de información que domina en los grupos G4 y G5 (no te informan, te tienes que tragar, te dan las noticias). Se revela una creencia compartida: hay que saber moverse por los medios y buscar. Es el único grupo que aprecia el valor regulador de contenidos que tiene la conducta interactiva del público a través de la red. Si bien -unos por experiencia propia y otros por lo que dicen ver en los demás- reconocen que este rol activo requiere una competencia determinada de las personas: preparación, experiencia, conocimientos, inversión de tiempo. Si no se dispone de esa competencia el castigo es que "te quedas atrás".

5. G4-Derechas despliega la dinámica conversacional de menor interacción de todos los grupos, acercándose más a una secuencia de turnos de palabra desconectados, no enlazados. Dedican más atención que ningún otro grupo a describir sus hábitos y preferencias: Ernesto: "coges y dices <yo quiero ver> y en casi todas las teles ya son smart tv y ya puedes poner Google y ver tu selección de contenidos que tú quieras, al final es más videoclub que autoservicio que tal...". Este es el patrón que regula el mercado de interés: "cada cual expone lo suyo". Sobre el presupuesto de que esa es la norma, cobra sentido una entrada como la de David, por ejemplo, que toma la palabra subrayando la distancia que (cree) que le separa de los demás David: "yo soy muy raro. Yo no veo la televisión casi nada o nada...". Esta dinámica de conversación replica la dinámica que los participantes perciben como dominante en el ecosistema: cada medio va a lo suyo y cada persona elige como quiere. Resulta coherente con ello que nunca nadie hable de "nosotros" como sujeto de una acción compartida y que, a diferencia de lo que ocurre en tres de los otros cuatro grupos, no aparezca nunca la idea de regulación del ecosistema.

6. G5-Sin estudios establecen un marco de diálogo de reconocimiento continuado de valores y argumentos de otros: "estoy de acuerdo con ella", "lo que ustedes han dicho". La consonancia se da principalmente en señalar que el sesgo informativo lo sufren todos los medios: "claro, lo que hemos dicho antes, respecto a que cada uno acerca la brasa a su sardina" (José Luis). Es un discurso dominado por el registro descriptivo y valorativo, donde se nombra a las noticias como objetos que existen por sí mismos (los medios se las dan al público, o "las cogen de agencias"), por lo que la tarea de quienes son más conscientes de la demanda de los medios es seleccionarlas. En esa tarea, señalan con consenso que los periodistas tienen que hacer lo que les dicen "los que mandan". No entran en confrontaciones. Sí aparece el nosotros, como sujeto y como objeto. Y se reconocen copropietarios del SPT (se amplía más adelante).

\subsection{Mapa de la economía temática de la atención}

La Tabla II presenta los cuatro temas más tratados por los grupos de acuerdo con los criterios metodológicos: función de los medios, control del ecosistema, los periodistas ${ }^{3}$ y la televisión pública ${ }^{4}$.

Observaciones sobre el mapa de economía temática:

1. Intensidad vertical: dos temas presentan hegemonía argumental en el sistema de discursos: 1) que los medios están controlados por intereses políticos y económicos que dominan su producción y 2) que los periodistas no son libres. Y se acompañan de expresiones valorativas de preocupación -es gravísimo dice Iñigo en G2-. En ocasiones se vincula a la necesidad de regular los medios.

2. Regulación: no entra en la tabla porque G5 sólo lo trata de forma indirecta y no tiene presencia en G45. Pero G1, G2 y G3 lo abordan con detalle.

3 Usan este término para referirse indistintamente a periodistas, presentadores, tertulianos, colaboradores.

4 No hablan de servicio público televisivo, salvo para referirse a la falta de definición del concepto servicio público.

5 En G4 nunca se menciona nada relacionado con reglas, normas, leyes o regulación. 
Tabla II.

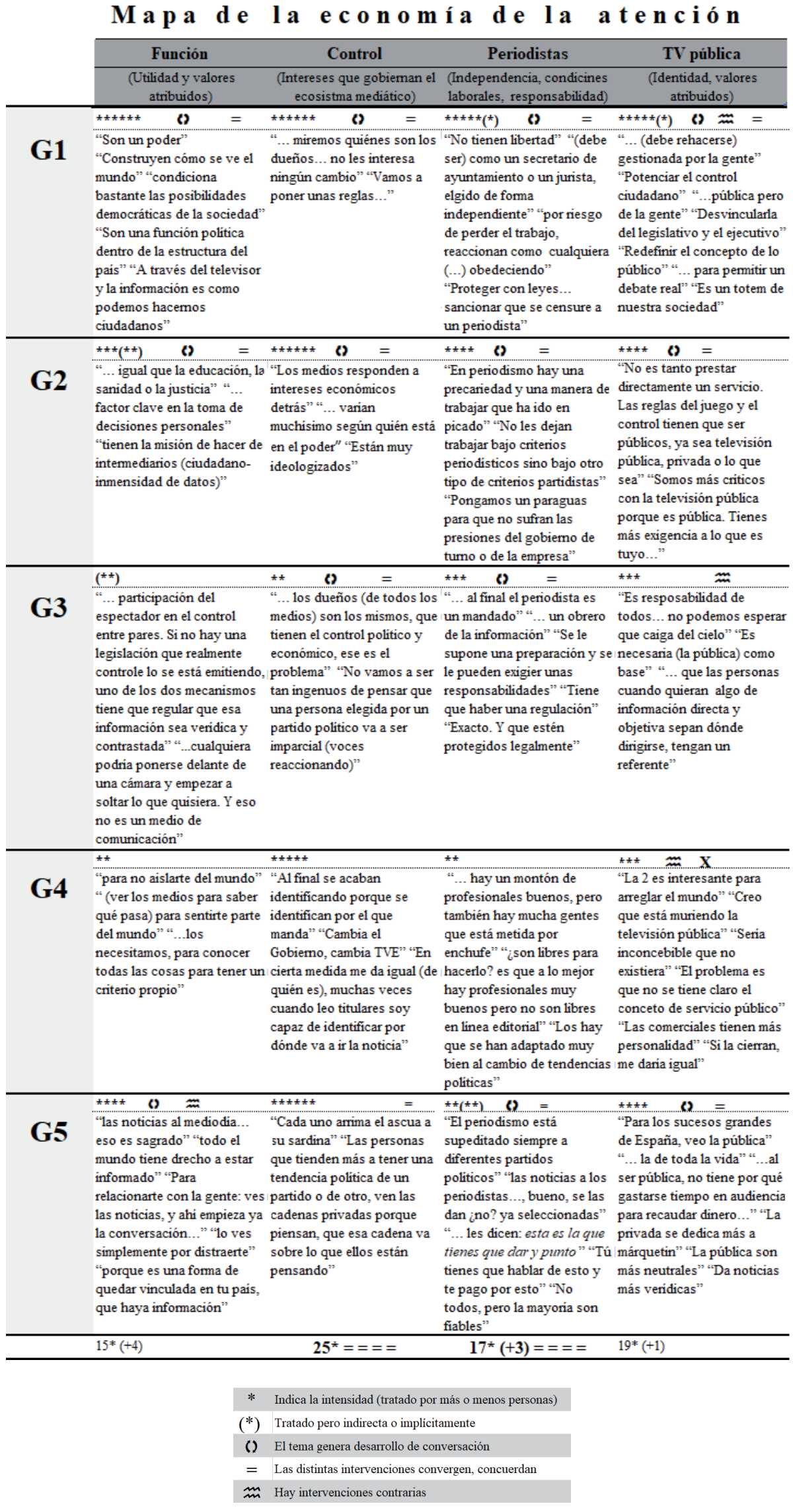


Regular para proteger a los periodistas (GlG2). Iñigo (G2): "dejémoslo un poco a los profesionales trabajar sin cortapisas y pongamos un paraguas para que no sufran las presiones del gobierno de turno o de la empresa de turno o del poder... y que ellos tengan que rendir cuenta ante los ciudadanos que son los que les pagan el sueldo y no ante los poderes que están más arriba".

Regular para evitar contenidos viciados (G1G2-G3-G5). Esteban (G3): "es necesario una regularización de todos los medios de comunicación, porque hay que diferenciar un poco entre libertad de expresión y libertad de blasfemia, (...) que se diga cualquier cosa. Esa libertad de expresión yo no la quiero porque eso no promueve valores que fomenten la ciudadanía. Yo quiero información detallada, contrastada, científica, no quiero una opinión como otra cualquiera. Entonces en ese sentido sí estoy de acuerdo en que tiene que haber, por mucho que me pese, pero también creo que en lo privado tiene que haber una pequeña regulación de unos mínimos. Tiene que haber unos mínimos y no todo puede valer, esa es mi opinión."

3. La financiación de la televisión pública aparece en todos los grupos, pero como apunte o acotación, no genera hilo argumental. El pago indirecto a las televisiones privadas es totalmente obviado. La conciencia de estar sosteniendo económicamente a una cadena de televisión solo se vincula con el SPT, y en dos perspectivas diferentes:

a) Negativa. Es una "carga". María José (G4): "cuando yo pienso en cadena pública pienso en pagar impuestos, si ese dinero que yo tengo que pagar en impuestos para que luego se financie una cadena de televisión que lo único que me ofrece es contenido malo, que no tiene índices de audiencia suficiente, pues sí, te da un poco de pena".

b) Positiva. Somos "copropietarios", nos vincula. Subyace un imaginario colectivo al que el hablante queda unido por un sentimiento de pertenencia (G1-G2-G4-G5).

Juani (G5): "Lo público es dinero de todo el mundo y no se debe dar ni para un lado ni para otro".
José Luis (G5): "todos nosotros con nuestros impuestos y eso, estamos contribuyendo a la televisión pública...".

Extracto de G5:

Julián: "Además que, es nuestro, que es de todos".

Victoria: "Exactamente que es de todos, lo pagamos entre todos y...".

José Luis: "Yo creo que sî".

Aparece una referencia a una colectividad histórica, que refleja una idea de patrimonio intergeneracional (heredado), cuan dicen que se ve "de toda la vida" o que si no existiera Ernesto (G4): “... esto es inconcebible, que no exista la televisión pública en España, ha costado mucho trabajo durante toda la vida, ha costado mucho trabajo, y no verlo...".

\subsection{Isotopías de la hostilidad mediática y de la inseguridad informativa}

La producción transversal de sentido en el conjunto de los discursos de todos los grupos se ha explorado mediante la localización de isotopías. Se ha operado sobre el concepto de isotopía del que parte Rastier (2005:111) tomado de Greimas: "un haz de categorías semánticas redundantes, subyacentes al discurso considerado". De acuerdo con Bueno y Blanco (1980:36): "La redundancia de determinados núcleos sémicos al interior del mismo discurso da por resultado una base permanente de referencias, en virtud de las cuales el discurso se hace coherente, o dicho de otro modo, habla de la misma cosa."

Se han localizado los enunciados con los que los participantes se refieren el ecosistema mediático. Se ha observado qué elecciones que realizan sobre el paradigma (elecciones entre el catálogo de opciones para un determinado sustantivo, verbo, adjetivo, adverbio). Y se ha buscado las que remiten a un mismo referente.

Antes de presentar todo el conjunto, se ilustra a continuación la aplicación de este planteamiento a un caso isotópico particular: la dificultad de identificar al sujeto emisor.

Ernesto (G4) dice: “... ¿quién es ese oscuro "decididor" que dice bueno la televisión pública va a tener un programa que se llama Masterchef?" Para describir al sujeto que decide la programación, este 
hablante ha elegido el término "oscuro". El remitente elegido es de identidad desconocida, cuerpo no claro, alguien que permanece oculto.

Carlos (G1): "Es que no solo los generadores de opinión, no son periodistas en muchísimos casos, sino que en otros son hasta anónimos.". Julia (G5): "Por internet porque al no haber noticias, noticias, físicas, vamos". Laurette (G3): "siempre la cabeza visible es alguien que está puesto a dedo ahí, persiguiendo determinados intereses y luego, vamos a intentar que no se note mucho".

Los cuatro están expresando un malestar vinculado a un ocultamiento. Su disconformidad elige términos -oscuro, anónimo, no físico, cabeza visible- que remiten a un mismo topo: cuerpo no visible, falta de corporeidad. Dificultad para reconocer. El corolario de esta isotopía es la desconfianza. Junto a ella, se han localizado otras cinco, que pasamos a describir.

\section{Isotopía del emisor opaco (Desconfianza)}

No se puede estar seguro de lo que un medio te dice porque desconoces quién te habla, su identidad, su formación, su currículum. Ernesto (G4) dice que cada cual lo que hace es "dar una imagen". Juan (G3): "nadie te filtra nada de si tiene titulación profesioal o no". Nacho (G4): “... intereses empresariales y además intereses que pueden tener un grupo de comunicación privado que está en manos de otro grupo que a su vez está en manos de un señor...".

\section{Isotopía de los intereses ocultos (Inseguridad)}

Aparece en todos los grupos. Explícitamente. Nacho (G5): "es que ya más que política, lo medios, privados se rigen más por el comercio y por quien los financia y, dependiendo de lo que a ellos les importe". Marichu (G2): “... responden a intereses económicos detrás (...) Entonces es un poco, porque claro detrás de cada noticia que sale y detrás de cada cadena hay diferentes discursos".

E implícitamente: "hipócritas", "son marionetas" (Manuela G1), o con el uso de adverbios como "aparentemente", "supuestamente"; otras veces, con construcciones como "dan la sensación de que están pagados por alguien" (Javier G1), "se deben a otros poderes, que no tienen nada que ver con los poderes ciudadanos" (Javier y Manuela G1), "me dan la impresión de" (María G4); "se les ve venir de lejos"
(Marichu, G3), "se ve el plumero" (Jesús, G2).

Esos intereses no confesados se perciben además como formando parte un entramado: el ecosistema de medios tiene detrás una estructura de intereses interconectados de modo inextricable. Términos de esta isotopía: engranaje, hibridación, travestismo, mezcla, puzle, intermediario, el "disfraz de lo digital", todo está medido y psicológicamente estudiado, no puede ser casualidad, todo está tremendamente coordinado. José Luis (G5) "cada uno arrima la brasa a su sardina".

- Julián (G5) “... pero políticamente Telecinco, pos, pos será de Berlusconi, Telecinco es Italiana, ehh, averigua tú lo que hay en Italia, pues el interés que crees que hay en Italia pues ese es el interés de ellos, y punto".

\section{Isotopía del engaño (Inseguridad)}

Para referirse a lo que cuentan los programas informativos, hablan de manipular, camuflar, ocultar, contaminar, te meten ficciones, show, distraerte, contenido seleccionado y acotado, margen controlado para hablar, no poder ver los intestinos, pasar de puntillas por los asuntos. Alba (G2) “... también probablemente se escoja una parte de los datos y se oculte la otra". Gustavo (G4) se lamenta usando el término detector ("no tengo un detector de eso"), que desvela la sospecha de que hay algo que no es patente. $\mathrm{Y}$ otras personas advierten de que el público se ve obligado a comprobar la información.

Un cuadro de referencias a que el contenido se desvía o no llega a la verdad (lo real, suelen decir).

- Javier (G1): "A mí me parece que de todos los objetivos que puede tener un medio de comunicación de información, probablemente hoy día el menos importante sea transmitir una información veraz (7:57) (...) tienen otros objetivos. Entre los objetivos es crear imaginarios, es manipular eh... a los ciudadanos, es distraer a los ciudadanos, es crear opinión...".

- Diego (G1): “... necesitaríamos que se mostrara... los intestinos... el interior, el qué se ha dejado de lado, el qué no se ha dejado".

- Gustavo (G4): “...me siento, eso contaminado, manejan el discurso para llevarte hacia un determinado sitio".

- Victoria (G5): "porque sí es verdad que toda la información que percibimos no es la que es real". 
- José Luis (G5): Una cosa es lo que se ve y otra cosa es lo que es

\section{Isotopía de guerra y caza (Riesgo, peligro)}

La descripción del ecosistema mediático como paisaje hostil, de peligros, aparece en todos los grupos, asociado a una isotopía de guerra, caza, amenaza, captura, batalla.

Dicen en G3: "tenemos muchas noticias, pero todo el mundo puede estar de acuerdo en que muchas noticias no están contrastadas. Y hay que tener mucho cuidado. Coincido, a raíz de lo que dijiste antes. Si es verdad que tenemos muchísima más capacidad para poder elegir, para poder optar por una u otra opción. Pero creo que no estamos educados todavía... porque es una formación constante de... el saber seleccionar. Y eso es la batalla que estamos...".

Representando a las noticias como sujeto dotado de voluntad (persecutoria): "te invaden" Lea (G1) "te encuentran a ti -dice Carlos (G1)- no tú a ellas".

Evocando indefensión, Alicia (G2): "te dejan en manos de empresas. Pues te sientes un poco como dejado en manos de fieras ¿no? De víboras.” Javier (G1) habla de atrapar y Manuela (G1) de situaciones de las que "es complicado salir". En G5 hablan de bombardeo informativo, de hundimiento, de dejarte acobardado; Clara (G5) dice "no te dejan levantar cabeza"; y cuando tocan el tema de los "programas de esos de cotilleo", Julián (G5) dice que la gente se mete en ellos para refugiarse de los informativos. Nacho (G4), cuando hablan de si no existiera la televisión pública, dice sería como "abrir la veda ya".

\section{Isotopía del desbordamiento (Indefensión)}

El ecosistema mediático se representa como territorio saturado en el que hay que elegir 6 . Jesús (G2): "pensamos que cuanto más información menos engaño, a lo mejor llega un momento que tienes tanta que no sabes dónde elegir". Esa sobrecarga que obliga a una labor ineludible de filtrado y selección es referida mediante un campo semántico de desmesura (son millones de opinadores), como algo inabordable: Carlos (G1): "O sea, ¿quién tiene capacidad de poder absorber todo eso? Lo que va a venir de la televisión es más

6 Excepto en G5. Este grupo no se plantea este problema porque domina un patrón receptivo de consumo de información: los medios te dan las noticias. información, mogollón, mogollón, saturación, pum pum pum, medios de comunicación... algo imposible de poder asumir." Javier (G1): "Uno no tiene ni el tiempo ni los recursos para poder saber de todos los medios... no damos para más". Marichu (G2): "a nivel individual es difícil que te pongas a manejarlos tú."

En G4: "no sabes dónde elegir", "exceso de información", "copan la atención". En G2: "capacidad de absorber", "volumen de datos".

Frente a esa desmesura, se estima necesario una ayuda, a veces identificado manifiestamente como intermediario, un referente. Y otras, implícito en el verbo elegido: manejo, criba, hacer una sintesis.

- Iñigo (G2): "Yo creo que esto lo hemos dicho, que como una interpretación de la realidad que te pueda orientar, ...en ese futuro de caos y de muchos inputs y tal... a mí me gustaría pensar que TVE se puede diferenciar... lo que es verdad que ahora hay ochocientos mil inputs y al final, no sabes qué creer y tal".

La isotopía del desbordamiento la completan las referencias a las formas de desenvolverte en ella: dedicar tiempo, saber moverte, saber buscar, hacer el esfuerzo, tener ciertas destrezas, estar documentado, incluso dedicarse a veces a hacer limpieza de suscripciones, porque Nacho (G4): "una saturación tan tremenda que llega un momento en el que no te informas te produce todo lo contrario, porque te inunda por completo".

- Esteban (G3) expone que la tarea de saber seleccionar nos afecta absolutamente a todos, tanto a un catedrático de ciencias de la información como a un campesino.

\section{Isotopía de la falta de calidad (Desorden informativo)}

Esta isotopía incluye más intervenciones y expresiones que ninguna otra. Y desencadena interacciones. Se distinguen dos tipos de hilos de conversación sobre calidad. Uno con foco sobre el producto, atendiendo a dos rasgos: que la información tenga interés (para mí) y objetividad-neutralidad. Y otros, focalizados sobre el proceso productivo: se contrasta poco, perversión, no hay checking, no hay barrera, se escoge una parte de los datos, las fuentes no son trazables, se monta un show. Esta división entre foco en el producto y foco en el proceso para crear al producto coincide con otra división, creencia y no creencia en que la 
objetividad es posible. Y a su vez, ambas se mantienen sobre dos estilos expresivos. Quienes hablan de foco en el producto y creencia en la objetividad usan preferentemente una construcción gramatical pasiva: "los medios te informan", o "te dan noticias". El otro perfil expresivo opta por la forma activa: me informo. Por último, este discurso con foco sobre proceso y con forma activa es sostenido distintivamente por quienes demandan que se establezcan y cumplan normas de procedimiento, que los medios contribuyan a la democracia y que garanticen participación. Reclamos que no aparecen cuando quienes hablan organizan su discurso centrándose en la satisfacción de necesidades $\mathrm{o}$ intereses personales.

\section{CONCLUSIÓN}

Las seis isotopías detectadas en el discurso del público sobre el ecosistema mediático y el papel del SPT arrojan una percepción de desorden informativo estructural en el sistema.

Respecto a la primera pregunta, sobre la identidad y función percibidas del SPT en el conjunto del sistema de medios, se define un reclamo de un servicio que sirva de referencia frente a un entorno descrito como inseguro, hostil y peligroso, de difícil manejo o navegación. Esta apreciación responde principalmente al flujo desbordante de contenidos y una falta de garantías sobre los intereses que mueven a los emisores. Se estima que estos emisores están sometidos a intereses políticos y corporativos poco transparentes.

Este discurso del público ratifica los diagnósticos académicos que se han descrito en el planteamiento de este texto. Entre las reacciones a este cuadro, emergen actitudes de evitación y sentimientos de rechazo emocional de la información, también detectados por otros trabajos (Newman et al., 2019: 25).

La creencia compartida de que circula un flujo elevado de información manipulada anima dos líneas de convicción: 1) requisito de la competencia mediática personal: las personas han de desarrollar una competencia personal específica para desenvolverse en el ecosistema mediático con seguridad y 2) requisito del marco regulador: las "reglas del juego" con las que actúan los mediadores han de ser fijadas. Ambos aparecen en el discurso del público como objetivos pendientes.
Los medios en general, y el SPT en particular, son percibidos como una función de potencia democrática, pero de ejecución deficiente e insatisfactoria. Una tensión que vienen señalando autoras como Fenton (2018). También se ratifican los principios ya asentados en la investigación sobre calidad informativa que encontramos compendiados en trabajos recientes como el de Romero-Rodríguez y Aguaded (2018): los usuarios del SPT manejan un discurso consciente de que ciertos valores de la calidad de la información son pre-informativos, derivan de la estructura sociopolítica y económica.

Respecto a la segunda pregunta de investigación -valores y rasgos que se le asignan al SPT- el discurso ciudadano discute aspectos que renuevan los marcos académicos tradicionales de comprensión del SPT, a veces confirmando y ampliando conclusiones de estudios más recientes:

1. La preocupación por la fiabilidad o/y calidad de los contenidos de los medios no se refiere únicamente el producto final, hay perfiles de público sensible a la calidad del proceso de producción. Concordancia con Larsen (2014:74).

2. La demanda de reconfiguración del SPT ya no se formula en términos de escala cuantitativa: más pluralismo, más acceso, más representación de voces o cultura, más diversidad, más inclusión... Se ha activado también una demanda de otro concepto de lo público. No solo al margen de los partidos políticos, sino como exigencia de otro concepto de ciudadanía y de gestión de lo común. Otra forma de ejercer la propiedad ciudadana del SPT se considera pendiente. Y otra forma de escuchar al público, en sintonía con lo que advierte Cullinane (2017:84) y de incorporarlo a la estructura del SPT, como marcan Lazer et al. (2018). Las investigaciones que exploran nuevos patrones y prácticas de producción televisiva en el SPT como los de Vanhaeght (2019) o Keinone $\&$ Klein (2017) concuerdan así a la perfección con las demandas presentes en el discurso del público aquí estudiado.

3. La necesidad de control y regulación (principalmente de procedimientos) articula una vertiente del discurso social. Se confirma la apreciación del informe de Reuters (Newman 
et al., 2018:19): el Estado es un sujeto de referencia para el orden del ecosistema mediático. La demanda de regulación consiguiente es más precisa en el discurso de quienes son más conscientes de la demanda la importancia del proceso de producción en la calidad de los contenidos informativos y de entretenimiento. Se plantea una tensión entre derecho a la libertad de expresión y a la protección frente a la intoxicación informativa. Más que nueva regulación, a veces se plantea desregular lo previamente regulado a favor del mercado, como ya sugirió Fenton (2010b: 50).

La profesionalidad de los periodistas es abordada con preocupación compartida, aunque desde posiciones discursivas diversas En positivo, como know how: una periodista es considerada una especialista confiable. En negativo, un profesional es alguien contratado por otro, y esto implica dependencia y vulnerabilidad laboral, que son percibidas como aspectos que precisan regulación para recomponer el orden informativo.

Estos parámetros delimitan el marco de discusión social sobre la regulación:

- Garantizar la transparencia sobre los intereses corporativos que sostienen los medios.

- Marco regulador universal para todos los medios, activando mecanismos normativos de garantías informativas: códigos de calidad y competencia sancionadora a través de entidades independientes.

- Crear estructuras legales de protección de la profesión periodística (independencia editorial y seguridad laboral).

- Asegurar un reclutamiento de profesionales en el SPT no vinculado a la estructura de partidos, más transparente, profesional y basado en méritos. (Se plantean como referentes el Poder Judicial, la Sanidad Pública, la Educación Pública).

- Ampliar la información accesible sobre los emisores: tanto sobre criterios y procesos de selección que han superado, como sobre formación y trayectoria profesional y curricular, tanto de profesionales de producción y creativos, como de gestores; tanto de medios públicos como privados.

\section{REFERENCIAS BIBLIOGRÁFICAS}

Arbona Ponce, P. (1994). El serial clásico de la BBC: la adaptación literaria como servicio público. En Eguiluz, F., Merino, R., Olsen, V., Pajares, E. y Santamaria, J., Trasvases culturales. Literatura, cine, traducción 1, 87-93. Bilbao: UPV.

Arriaza Ibarra, K.; Nowak, E. and Kuhn, R. (2015). Introduction: the relevance of Public Service Media in Europe. En Arriaza Ibarra, K.; Nowak, Eva and Kuhn, R. (eds.), Public Service Media in Europe: A Comparative Approach (pp. 1-8). New York: Routledge.

Bakshy, E., Messing, S. \& Adamic, LA. (2015). Exposure to ideologically diverse news and opinion on Facebook. Science 348 (6239), 1130-1132. https://doi:10.1126/ science.aaal160

Balaban, D. \& Mustaeta, M. (2019). Users'Perspetive on the Credibility of Social Media Influencers in Romania and Germany. Romanian Journal of Communicatin and Public Relations, 21, no. 1 (46), 31-46. https://doi: 10.21018/ rjcpr.2019.1.269

Bardoel, J. \& d'Haenens, L. (2008). Reinventing public service broadcasting in Europe: prospects, promises and problems. Media, Culture \& Society, 30 (3), 337-355. https://doi: 10.1177/0163443708088791

BBC (2019): BBC Transparency Review

Blanco, D. y Bueno, R. (1980). Metodología del análisis semiótico. Lima: Universidad de Lima.

Blas Arroyo, JL. (2004). Sociolingüística del español. Madrid: Cátedra.

Blumler, JG. (ed.) (1993): Televisión e interés público. Barcelona: Bosch.

Bourdon, J., Buchman, MM. \& Kaufman, PB. (2019). Editorial: Public Service Broadcasting in the Digital Age. VIEW Journal of European Television History \& Culture, 8 (16), 1-4. https://doi: 10.18146/2213-0969.2019. jethc 170

Briz, A. (2001). El español coloquial en la conversación: esbozo de pragmagramática. Barcelona: Ariel Lingüística.

Callejo, J. (2000). El grupo de discusión: introducción a una práctica de investigación. Barcelona: Ariel.

Campos-Freire, F., Túñez-López, M. y Rodríguez-Castro, M. (2018). Retos 2020-30 para el servicio audiovisual público. En Rodríguez-Castro, M., Pérez-Seijo, S. y Campos-Freire, F. (Eds.), La TV pública en Europa: Innovación, Retos y Tendencias (9-22). Cuadernos Artesanos de Comunicación, cac 146. La Laguna (Tenerife): Latina. https://doi: 10.4185/cac146.

CIS (2019). Postelectoral Elecciones autonómicas y municipales 2019. Estudio 3253. Junio-julio 2019.

Collins, R., Finn, A., Mcfadyen, S. y Hoskins, C. (2001). Public Service Broadcasting Beyond 2000: Is There a Future for Public Service Broadcasting? Canadian Journal of Communication 26, 3-15. https://doi.org/10.22230/ cjc.2001v26nlal192 
Conde Gutiérrez del Álamo, F. (2010). Análisis sociológico del sistema de discursos. Madrid: CIS. Cuadernos Metodológicos, 43.

Conway, K. (2009). Public Service Broadcasting and the Failure of Political Representation. Velvet Light Trap, Fall 2009, 64-75. https://doi:10.5555/vlt.2009.64.64

Council of Europe (1994). The media in a Democratic society. Political Declaration, Resolutions and Statement, 4th European Ministerial Conference on Mass Media Policy.

Council of Europe (2004). Public Broadcasting Service Recommendation 1641, 27 January.

Cushion, S. (2012). The Democratic Value of News: Why Public Service Media Matter. Palgrave Macmillan: Basingstoke and New York.

Dahlgren, P. (2009). Media and political engagement: citizens, communication and democracy. Cambridge University Press.

Department for Culture, Media \& Sport (2016). A BBC for the future: a broadcaster of distinction. London: Crown.

Department for Culture, Media \& Sport (2015). BBC Charter Review. Public consultation July-October 2015. London: Crown.

Elías, C. (2018). Fakenews, poder y periodismo en la era de la posverdad y <hechos alternativos $>$. Ámbitos, 40, 1-6. https://doi.org/10.12795/Ambitos.2018.i40.04

Fairlouhg, N. (2006). Discourse and Social Change. Cambridge: Polity Press.

Fairlough, N. (1993). Critical Discourse Analysis and the Marketization of Public Discourse: The Universities. Discourse \& Society, 4 (2), 133-168. https:// doi:10.1177/0957926593004002002

Fenton, N. (2018). Fake Democracy: The Limits of Public Sphere Theory. Javnost: The Public, 25 (1-2), 28-34. https://doi: 10.1080/13183222.2018.1418821

Fenton, N. (2010a) (ed.). New Media, Old News: Journalism and Democracy in the Digital Age. London: Sage

Fenton, N. (2010b). New Media, Journalism and Democracy: Figments of a Neo-Liberal Imagination? Media E Journalism, 17 (9), 41-52.

Fernández, Á.N. (1995). Estructura lógica de las actitudes proposicionales. Philosophica Malacitana. Suplemento III "Filosofía y Ciencias Cognitivas" (pp. 97-109).

Freedman, D. (2019). "Public Service" and the Journalism Crisis: Is the BBC the Answer? Television \& New Media, 20 (3), 203-218. https://doi:10.1177/1527476418760985

Freedman, D. (2016a). A Future for Public Service Television: Content and Platforms in a Digital World. Project Report. Goldsmiths, University of London. http://futureoftv.org. uk/report/

Freedman, D. (2016b). The BBC and public service TV has a future - but it must change to survive. The Coversation, $6^{\text {th }}$ uly, 2016.

Gallardo Paúls, B. (1996). Análisis conversacional y pragmática del receptor. Valencia: Episteme.

Gardner, S. (2017). Public Broadcasting Its past and Its Future. Knight Foundation.

Garnham, N. (1983). Public Service versus the Market. Screen 5 (1), 6-28. https://doi.org/10.1093/screen/24.1.6
Goffman, E. (1967). Interaction Ritual / Essays on Face to Face Behaviour. New York: Pantheon Books.

Goffman, E. (1956). The Presentation of self in Everyday Life. Univesity of Edinburgh, Social Science Research Centre. Monogaph 2.

Gordo, A.J. y Serrano, A. (Coords.) (2008). Estrategias y prácticas cualitativas de investigación social. Madrid: Pearson-Prentice Hall.

Gutiérrez Brito, J. (2014). Dinámica del grupo de discusión. Madrid: CIS

Halliday, M.A.K. (2013). El lenguaje como semiótica social. La interpretación social del lenguaje y el significado. México: Fondo de Cultura Económica

Haraway, D.J. (1995). Ciencia, ciborgs y mujeres. La reinvención de la naturaleza. Madrid: Cátedra.

Humphreys, P. (2008). Redefining Public Service Media: A Comparative Study of France, Germany and the UK. En 4th Annual RIPE Conference, 'Public Service Media in the 21th Century: Participation, Partnership and Media Development. October 9-11, 2008. Mainz, Germany.

Holton, A. \& Chyi, H.I. (2012). News and the Overloaded Consumer: Factors Influencing Information Overload Among News Consumers. Cyberpsychology, Behavior, and Social Networking, 15 (11), 619-624. https://doi:10.1089/ cyber.2011.0610

Ibáñez, J. (2015). Cómo se realiza una investigación mediante grupos de discusión. En García Ferrando, Manuel et al. (comps.). El análisis de la realidad social. Métodos y técnicas de investigación (pp. 418-434). Madrid: Alianza Editorial.

Ipsos Mori (2017). BBC Distinctiveness. June, 2017.

Kalsnes, B. (2018). Subject: Journalism Studies, Media and Communication Policy in Oxford Research Encyclopedia, Communication, Sep 2018. https://doi:10.1093/ acrefore/9780190228613.013.809

Keinonen, H. \& Klein Shagrir, O. (2017). From Public Service Broadcasting to Soci(et)al TV. Nordicom Review 38 (1), 65-79. https://doi:10.1515/nor-2016-0037

Larsen, H. (2014). The Legitimacy of Public Service Broadcasting in the 21 tf Century. Nordicon Review, 35 (2), 65-76. https://doi.org/10.2478/nor-2014-0015

Lazer, DMJ., Baum, MA., Benkler, Y., Berinsky, AJ., Greenhill, KM. (2018). The science of fake news. Science, 359(6380), 1094-1096. https://doi:10.1126/science.aao2998The science of fake news

Lamuedra, M., Mateos, C. \& Borullón-Lozano, M. (2020). The role of public service journalism and television in fostering public voice and the capacity to consent: An analysis of Spanish viewers' discourses. Journalism, 21 (11), 1798-1815. https://doi: 10.1177/1464884919847593

Lamuedra, M., Mateos, C. \& Borullón-Lozano, M. (2019). Normative and Audience Discourses on Public Service Journalism at a "Critical Juncture": the Case of TVE in Spain. Journalism Studies, 20 (11), 1528-1545. https://doi $: 10.1080 / 1461670 X .2018 .1528880$

Lee-Wright, P. (2010). Culture Shock: New media and organizational change in the BBC. En Fenton, N. (ed.), New Media, Old News: Journalism and Democracy in the Digital Age. (pp. 71-86). London: Sage. 
Levi, S. (2019). \#Fake You / Fake News y desinformación. Barcelona: Rayo Verde.

Meijer, I. C. (2012). Valuable journalism: A search for quality from the vantage point of the user. Journalism: Theory, Practice \& Criticism, 14(6), 754-770. https:// doi:10.1177/1464884912455899

Moe, H. (2010). Defining public service beyond broadcasting: the legitimacy of different approaches. International Journal of Cultural Policy 2010, 1-17, iFirst Article. https:// doi.org/10.1080/10286630903049912

Newman, N., Fletcher, R., Kalogeropoulos, A., Levy, D. y Nielsen, RK. (2018). Reuters Institute Digital News Report 2018. Reuters Institute $\&$ University of Oxford.

Newman, N., Fletcher, R., Kalogeropoulos, A., Levy, D. y Nielsen, RK. (2019). Reuters Institute Digital News Report 2019. Reuters Institute \& University of Oxford.

Nissen, C.S. (2006). Public Service Media in the Information Society. Strasbourg (France): Council of Europe's Group of Specialists on Public Service Broadcasting in the Information Society.

Nordicity, April 2016. Analysis of Government Support for Public Broadcasting.

Nordicity, April 2011. Analysis of Government Support for Public Broadcasting and Other Culture in Canada.

Ofcom (2020). Covid-19 news and information: consumption and attitudes. Results from week one of Ofcom's online survey. 9/04/2020

Ofcom (2018). Public service broadcasting in the digital age. Supporting PSB for the next decade and beyond. 8/03/2018

Padovani, C. \& Tracey, M. (2003). Report on the Conditions of Public Service Broadcasting. Television \& New Media, 4 (2), 131-153. https://doi.org/10.1177/1527476402250677

Peirano, M. (2019). El enemigo conoce el sistema. Barcelona: Debate.

Peñamarín, C. (2014). Esfera pública y construcción del mundo común. El relato dislocado. CIC Cuadernos de Información y Comunicación, 19, 103-124. https:// doi:10.5209/rev_CIYC.2014.v19.43906

Pew Research Center (2018). In Westernn Europe, Public Attitudes Toward News Media More Divided by Populist Views Than Left-Right Ideology. Washington.

Pew Research Center (2005). Trends 2005. Washington.

Raboy, M. (2006). Creating the Conditions for Communication in the Public Good. Canadian Journal of Communication, 31 (2), 289-306. https://doi. org/10.22230/cjc.2006v31n2al733

Rastier, F. (2005 [1987]). Semántica interpretativa. México D.F: Siglo XXI Editores.

Romero-Rodríguez, Luis M. y Aguaded, Ignacio (2017). Toward a taxonomy of newspaper information quality: An experimental model and test applied to Venezuela dimensions found in information quality. Journalism, 18 (19). 1327-1345. doi.org/10.1177/1464884916663596

Shao, C., Hui, PM., Wang, L., Jiang, X., Flammini, A., Menczer, F. \& Ciampaglia, JL. (2018). Anatomy of an online misinformation network. PLOS ONE, 13(4), e0196087. https://doi:10.1371/journal.pone.0196087
Sahragard, R. \& Davatgarzadeh, G. (2010). The Representation of Social Actors In Interchange Third Edition Series: A Critical Discourse Analysis. JTLS Journal of Teaching Language Skills, Vol. 29 (1), 67-89. https://doi: 10.22099/jtls.2012.401

Scannell, P. (1989). Public service broadcasting and modern public life. Media, Culture \& Society, 11(2), 135-166. https://doi:10.1177/016344389011002002

Sehl, A., Cornia, A. \& Nielsen, R.K. (2016). Public Service News and Digital Media. Reuters Institute.

Steemers, J. (2001). In Search of a Third Way: Balancing Public Purpose and Commerce in German and British Public Service Broadcasting. Canadian Journal of Communication, 26, 69 - 87. https:// doi.org/10.22230/ cjc.2001v26n1al196

Vanhaeght, A-S. (2019). Audience Participation in PSM from a Media-centric to a Society-centric Approach: The Monitor as a Best Practice of the Dutch Public Broadcaster NPO. VIEW Journal of European Television History and Culture, 8(16), 45-58. https://doi:10.18146/22130969.2019.jethc174

Van Dijk, T.A. (comp.) (2000). El discurso como interacción social. Barcelona: Gedisa.

Tuchman, G. (2002). Media institutions. Qualitative methods in the study of news. En Jensen, K. B., \& Jankowski, N. W. (Eds.), A Handbook of Qualitative Methodologies for Mass Communication Research (pp. 79-92). London and New York: Routledge.

Van Dijk, T.A. (1999). El análisis crítico del discurso. Anthropos, 186, 23-36.

Vosoughi, S., Roy, D. \& Aral, S. (2018). The spread of true and false news online. Science, 359 (6380),1146-1151. https://doi: 10.1126/science.aap9559

Waisbord, S. (2018). Truth is What Happens to News. Journalism Studies, 19 (8), 1866-1878. https://doi: 10.1080/1461670X.2018.1492881

Zallo, R. (2010). La política de Comunicación Audiovisual del gobierno socialista (2004-2009): un giro neoliberal. Revista Latina de Comunicación Social, 65, 14-29. https:// doi: 10.4185/RLCS-65-2010-880-014-029

\section{NOTA BIOGRÁFICA}

\section{CONCHA MATEOS}

Licenciada en Ciencias de la Información por la Universidad Complutense de Madrid (1990) y premio extraordinario de doctorado en Ciencias Sociales en La Laguna, Tenerife (2004). Profesora titular de Comunicación Audiovisual en la Universidad Rey Juan Carlos. Antes de dedicarse a la academia, ejerció como periodista durante una década en diversos medios de comunicación y gabinetes de prensa de organizaciones políticas. 
